\title{
Vitali sets and Hamel bases that are Marczewski measurable
}

\author{
by \\ Arnold W. Miller (Madison, WI) and \\ Strashimir G. Popvassilev (Sofia and Auburn, AL)
}

\begin{abstract}
We give examples of a Vitali set and a Hamel basis which are Marczewski measurable and perfectly dense. The Vitali set example answers a question posed by Jack Brown. We also show there is a Marczewski null Hamel basis for the reals, although a Vitali set cannot be Marczewski null. The proof of the existence of a Marczewski null Hamel basis for the plane is easier than for the reals and we give it first. We show that there is no easy way to get a Marczewski null Hamel basis for the reals from one for the plane by showing that there is no one-to-one additive Borel map from the plane to the reals.
\end{abstract}

Basic definitions. A subset $A$ of a complete separable metric space $X$ is called Marczewski measurable if for every perfect set $P \subseteq X$ either $P \cap A$ or $P \backslash A$ contains a perfect set. Recall that a perfect set is a non-empty closed set without isolated points, and a Cantor set is a homeomorphic copy of the Cantor middle-third set. If every perfect set $P$ contains a perfect subset which misses $A$, then $A$ is called Marczewski null. The class of Marczewski measurable sets, denoted by $(s)$, and the class of Marczewski null sets, denoted by $\left(s^{0}\right)$, were defined by Marczewski [10], where it was shown that $(s)$ is a $\sigma$-algebra, i.e. $X \in(s)$ and $(s)$ is closed under complements and countable unions, and $\left(s^{0}\right)$ is a $\sigma$-ideal in $(s)$, i.e. $\left(s^{0}\right)$ is closed under countable unions and subsets. Several equivalent definitions and important properties of $(s)$ and $\left(s^{0}\right)$ were proved in [10], for example every analytic set is Marczewski measurable, the properties $(s)$ and $\left(s^{0}\right)$ are preserved under "generalized homeomorphisms" (also called Borel bijections), i.e. one-to-one onto functions $f$ such that both $f$ and $f^{-1}$ are Borel measurable (i.e. pre-images of open sets are Borel), a countable product is in

2000 Mathematics Subject Classification: 03A15, 28A05, 54H05.

The second author was supported by the Bulgarian Foundation EVRIKA (I.3 IB85/22.07.1997, 02.09.1997) and by a GTA Fellowship from Auburn University. 
$(s)$ if and only if each factor is in $(s)$, and a finite product is in $\left(s^{0}\right)$ if and only if each factor is in $\left(s^{0}\right)$.

The perfect kernel of a closed set $F$ is the set of all $a \in F$ such that $U \cap F$ is uncountable for every neighborhood $U$ of $a$.

A set is totally imperfect if it contains no perfect subset. A totally imperfect set of reals cannot contain uncountable closed set, so it must have inner Lebesgue measure zero. A set $B$ is called Bernstein set if every perfect set intersects both $B$ and the complement of $B$, or, equivalently, both $B$ and its complement are totally imperfect. Clearly, no Bernstein set can be Marczewski measurable.

A set $A$ is perfectly dense if its intersection with every non-empty open set contains a perfect set.

Let $\mathbb{R}$ denote the set of all real numbers and $\mathbb{Q}$ denote the set of all rational numbers. We use $\mathfrak{c}$ to denote the cardinality of the continuum.

The linear closure (or span) over $\mathbb{Q}$ of a non-empty set $A \subseteq \mathbb{R}$ is the set

$$
\operatorname{span}(A)=\left\{q_{1} a_{1}+\ldots+q_{n} a_{n}: n<\omega, q_{j} \in \mathbb{Q}, a_{j} \in A\right\}
$$

and $\operatorname{span}(\emptyset)=\{0\} . A$ is called linearly independent over $\mathbb{Q}$ if $q_{1} a_{1}+\ldots+$ $q_{n} a_{n} \neq 0$ whenever $n<\omega, q_{j} \in \mathbb{Q}$ for $1 \leq j \leq n$ with $q_{j} \neq 0$ for at least one $j$, and $a_{1}, \ldots, a_{n}$ are different points from $A$. A linearly independent set $H$ such that $\mathbb{R}=\operatorname{span}(H)$ is called a Hamel basis. Note a Hamel basis must have cardinality $\mathfrak{c}$. The inner Lebesgue measure of any Hamel basis $H$ is zero (Sierpiński [8], see also Erdős [2]). A Hamel basis can have Lebesgue measure 0 (see Jones [4], or Kuczma [6], Chapter 11).

A Hamel basis $H$ which intersects every perfect set is called a Burstin set [1]. Every Burstin set $H$ is also a Bernstein set, otherwise if $P \subseteq H$ for some perfect set $P$, by the linear independence of $H$ it follows that $H \cap 2 P=\emptyset$ (where $2 P=\{2 p: p \in P\})$, a contradiction since $2 P$ is a perfect set.

A Burstin set can be constructed as follows. List all perfect subsets of $\mathbb{R}$ as $\left\{P_{\alpha}: \alpha<\mathfrak{c}\right\}$, pick a non-zero $p_{0} \in P_{0}$ and, using the facts that

$$
|\operatorname{span}(A)| \leq|A|+\omega<\mathfrak{c} \quad \text { if }|A|<\mathfrak{c}
$$

and $\left|P_{\alpha}\right|=\mathfrak{c}$ for each $\alpha$, pick by induction

$$
p_{\alpha} \in P_{\alpha} \backslash \operatorname{span}\left(\left\{p_{\beta}: \beta<\alpha\right\}\right)
$$

and let $H_{\mathfrak{c}}=\left\{p_{\alpha}: \alpha<\mathfrak{c}\right\}$. If $H$ is a maximal linearly independent set with $H_{\mathfrak{c}} \subseteq H$, then $H$ is a Burstin set.

A set $V \subseteq \mathbb{R}$ is called a Vitali set if $V$ is a complete set of representatives (or a transversal) for the equivalence relation defined by $x \sim y$ iff $x-y \in \mathbb{Q}$, i.e. for each $x \in \mathbb{R}$ there exists a unique $v \in V$ such that $x-v \in \mathbb{Q}$. No Vitali set is Lebesgue measurable or has the Baire property. One may construct a Vitali set which is a Bernstein set. 
Perfectly dense Marczewski measurable Vitali set. Recall that an equivalence relation on a space $X$ is called Borel if it is a Borel subset of $X \times X$. The Vitali equivalence $\sim$ as defined above is Borel. We first show that a Vitali set cannot be Marczewski null.

THEOREM 1. Suppose $X$ is an uncountable separable completely metrizable space with a Borel equivalence relation, $\equiv$, on it with every equivalence class countable. Then, if $V \subseteq X$ meets each equivalence class in exactly one element, $V$ cannot be Marczewski null.

P r o o f. By a theorem of Feldman and Moore [3] every such Borel equivalence relation is induced by a Borel action of a countable group. This implies that there are countably many Borel bijections $f_{n}: X \rightarrow X$ for $n \in \omega$ such that $x \equiv y$ iff $f_{n}(x)=y$ for some $n$. If $V$ were Marczewski null, then so would $X=\bigcup_{n<\omega} f_{n}(V)$.

To obtain a Marczewski measurable Vitali set we will use the following theorem:

THEOREM 2 (Silver [9]). If $E$ is a coanalytic equivalence relation on the space of all real numbers and $E$ has uncountably many equivalence classes, then there is a perfect set of mutually E-inequivalent reals (in other words, an E-independent perfect set). In the case of a Borel equivalence relation $E$, one can drop the requirement that the field of the equivalence be the whole set of reals.

If $E \subseteq X \times X$ is a Borel equivalence relation, where $X$ is an uncountable separable completely metrizable space, and $B$ is a Borel subset of $X$, then the saturation of $B,[B]_{E}=\bigcup_{x \in B}[x]_{E}$, is analytic since it is the projection onto the second coordinate of the Borel set $(B \times X) \cap E$. The saturation need not be Borel, for example let $B$ be a Borel subset of $X=\mathbb{R}^{2}$ whose projection $\pi_{1}(B)$ into the first coordinate is not Borel. Define $(x, y) E(u, v)$ iff $x=u$ (i.e. two points are equivalent if they lie on the same vertical line). Then $[B]_{E}=\pi_{1}(B) \times \mathbb{R}$ is not Borel. On the other hand, if $E$ is a Borel equivalence with each equivalence class countable, and $f_{n}$ are as in the proof of Theorem 1, then the saturation $[B]_{E}=\bigcup_{n<\omega} f_{n}(B)$ of every Borel set $B$ is Borel.

THEOREM 3. Suppose $X$ is an uncountable separable completely metrizable space with a Borel equivalence relation $E$. Then there exists Marczewski measurable $V \subseteq X$ which meets each equivalence class in exactly one element.

Proof. Let $\left\{P_{\alpha}: \alpha<\mathfrak{c}\right\}$ list all perfect subsets of $X$. We will describe how to construct disjoint $C_{\alpha}$, each $C_{\alpha}$ either countable (possibly finite or 
empty) or a Cantor set such that the set $V_{\alpha}=\bigcup_{\beta<\alpha} C_{\beta}$ is $E$-independent. Then extend the set $V_{\mathfrak{c}}=\bigcup_{\alpha<\mathfrak{c}} C_{\alpha}$ to a maximal $E$-independent set $V$.

CASE (a). If $P_{\alpha} \cap\left[C_{\beta}\right]_{E}$ is uncountable for some $\beta<\alpha$, then let $C_{\alpha}=\emptyset$.

Subcase (a1): $\left|P_{\alpha} \cap C_{\beta}\right|>\omega$. Then the perfect kernel of $P_{\alpha} \cap C_{\beta}$ is contained in both $P_{\alpha}$ and $V_{\alpha}$ (and hence in $V$ ).

Subcase (a2): $\left|P_{\alpha} \cap C_{\beta}\right|=\omega$. Then, since $P_{\alpha} \cap\left[C_{\beta}\right]_{E} \backslash C_{\beta}$ is uncountable analytic, it contains a perfect set $Q$ which misses $V$.

Case (b): Not Case (a). Then

$$
\left|P_{\alpha} \cap\left[V_{\alpha}\right]_{E}\right|=\left|P_{\alpha} \cap \bigcup_{\beta<\alpha}\left[C_{\beta}\right]_{E}\right| \leq|\alpha| \omega<\mathfrak{c},
$$

and hence $P_{\alpha} \backslash\left[V_{\alpha}\right]_{E}$ contains a Cantor set $P$.

Subcase (b1): The restriction of $E$ to $P$ has only countably many classes. Let $C_{\alpha}$ be a countable $E$-independent subset of $P$ with $P \subseteq\left[C_{\alpha}\right]_{E}$. Then $P \backslash C_{\alpha}$ contains a perfect set which misses $V$.

SubCASE (b2): Case (b) but not case (b1). Then, by the above theorem of Silver, there is a perfect $E$-independent set $C_{\alpha} \subseteq P$ (and $C_{\alpha} \subseteq V$ ).

REMARK 4. The Vitali equivalence shows that a Borel equivalence need not have a transversal that is Lebesgue measurable or has the Baire property. See Kechris [5], 18.D, for more on transversals of Borel equivalences.

THEOREM 5. There exists a Vitali set which is Marczewski measurable and its intersection with each non-empty open set contains a perfect set.

Proof. By Theorem 3 there is a Marczewski measurable Vitali set $V$, and by Theorem $1, V$ contains a perfect set $C$. Split $C$ into countably many Cantor sets $C_{0}, C_{1}, \ldots$, fix a basis $\left\{B_{n}: n<\omega\right\}$ for the topology of $\mathbb{R}$ and pick rational numbers $q_{n}$ so that the set $q_{n}+C_{n}=\left\{q_{n}+c: c \in C_{n}\right\}$ intersects $B_{n}$ for each $n$. Then

$$
V^{\prime}=(V \backslash C) \cup \bigcup\left\{\left(q_{n}+C_{n}\right): n<\omega\right\}
$$

is a perfectly dense Marczewski measurable Vitali set.

REMARK 6. A Vitali set $V$ cannot have the stronger property that its intersection with every perfect set contains a perfect set. This is because if $V$ contains a perfect set $P$, then the perfect set

$$
P^{\prime}=P+1=\{p+1: p \in P\}
$$

does not intersect $V$. Similarly, if $H$ is a Hamel basis that contains a perfect set $P$, then

$$
2 P=\{2 p: p \in P\}
$$

is a perfect set which misses $H$. 


\section{Marczewski null Hamel bases}

REMARK 7 (Erdős [2]). Under $\mathrm{CH}$ there is a Hamel basis $H$ which is a Lusin set (and hence Marczewski null). To see this, note that by a result of Sierpiński there is a Lusin set $X$ such that $X+X=\{x+y: x, y \in X\}=\mathbb{R}$ (see e.g. [7]). Let $H$ be any maximal linearly independent subset of $X$; then clearly $\operatorname{span}(H)=\operatorname{span}(X)=\mathbb{R}$.

Our construction (without $\mathrm{CH}$ ) of a Marczewski null Hamel basis is slightly simpler for the plane, so we do it first.

THEOREM 8. There exists a Hamel basis, H, for $\mathbb{R} \times \mathbb{R}$, i.e. a basis for the plane as a vector space over $\mathbb{Q}$, which is a Marczewski null set, i.e., every perfect set contains a perfect subset disjoint from $H$.

Lemma 9. Suppose $V$ with $|V|<\mathfrak{c}$ is a subspace of $\mathbb{R} \times \mathbb{R}$ as a vector space over $\mathbb{Q}$ (not necessarily closed $), p \in \mathbb{R} \times \mathbb{R}, y \in \mathbb{R}$, and

$$
U \subseteq U_{y}=(\{y\} \times \mathbb{R}) \cup(\mathbb{R} \times\{y\})
$$

with $|U|<\mathfrak{c}$. Then there exists a finite $F \subseteq\left(U_{y} \backslash U\right)$ with $p \in \operatorname{span}(F \cup V)$ and such that $F$ is linearly independent over $\mathbb{Q}$ and independent over $V$, i.e., $\operatorname{span}(F)$ meets $V$ only in the zero vector.

Proof. CASE 1: $p=(u, 0)$. Let $y_{1}$ and $y_{2}$ be so that

$$
y_{2}-y_{1}=u, \quad\left(y_{1}, y\right) \notin U \quad \text { and } \quad\left(y_{2}, y\right) \notin U .
$$

Clearly, $p \in \operatorname{span}\left(\left\{\left(y_{1}, y\right),\left(y_{2}, y\right)\right\}\right)$. Let

$$
F \subseteq\left\{\left(y_{1}, y\right),\left(y_{2}, y\right)\right\} \subseteq U_{y} \backslash U
$$

be minimal such that $p \in \operatorname{span}(V \cup F)$. Then $F$ works.

CAse 2: $p=(0, v)$. Obviously, this case is symmetric.

Case 3: $p=(u, v)$. Apply Case 1 to $(u, 0)$ obtaining $F_{1}$. Let

$$
V^{\prime}=\operatorname{span}\left(V \cup F_{1}\right)
$$

and apply Case 2 to $V^{\prime}$ obtaining $F_{2}$ (and let $F=F_{1} \cup F_{2}$ ) so that

$$
(u, 0),(0, v) \in \operatorname{span}\left(V \cup F_{1} \cup F_{2}\right) .
$$

Proof of Theorem 8. The theorem is proved from the lemma as follows. Let $\left\{B_{\alpha}: \alpha<\mathfrak{c}\right\}$ list all uncountable Borel subsets of $\mathbb{R} \times \mathbb{R}$ which have the property that for every $y$ the set $B_{\alpha} \cap U_{y}$ is countable. Let also $\left\{p_{\alpha}\right.$ : $\alpha<\mathfrak{c}\}=\mathbb{R} \times \mathbb{R}$ and $\left\{y_{\alpha}: \alpha<\mathfrak{c}\right\}=\mathbb{R}$. Construct an increasing sequence $H_{\alpha} \subseteq \mathbb{R} \times \mathbb{R}$ for $\alpha<\mathfrak{c}$ so that

1. $H_{\alpha}$ are linearly independent over the rationals,

2. $\beta<\alpha$ implies $H_{\beta} \subseteq H_{\alpha}$,

3. $H_{\lambda}=\bigcup_{\alpha<\lambda} H_{\alpha}$ at limit ordinals $\lambda$,

4. $H_{\alpha+1} \backslash H_{\alpha} \subseteq U_{y_{\alpha}}$ is finite, 
5. $p_{\alpha} \in \operatorname{span}\left(H_{\alpha+1}\right)$,

6. $H_{\alpha} \cap B_{\beta} \subseteq H_{\beta+1}$ whenever $\beta<\alpha$,

7. $H_{\alpha} \cap U_{y_{\beta}} \subseteq H_{\beta+1}$ whenever $\beta<\alpha$.

At successor ordinals $\alpha+1$ apply the lemma with $p=p_{\alpha}, V=\operatorname{span}\left(H_{\alpha}\right)$, and

$$
U=\left\{p \in U_{y_{\alpha}}: \exists \beta<\alpha\left(p \in B_{\beta} \text { or } p \in U_{y_{\beta}}\right)\right\} .
$$

Then let $H_{\alpha+1}=H_{\alpha} \cup F$.

The set $H=\bigcup_{\alpha<\mathrm{c}} H_{\alpha}$ is a Hamel basis; note that for every $y_{\alpha} \in \mathbb{R}$ we have $H \cap U_{y_{\alpha}} \subseteq H_{\alpha+1}$ and so

$$
\left|H \cap U_{y_{\alpha}}\right|<\mathfrak{c}
$$

and similarly for every $\alpha$ we have

$$
\left|H \cap B_{\alpha}\right|<\mathfrak{c} .
$$

To see that $H$ is Marczewski null, suppose that $P$ is any perfect subset of the plane. If $P \cap U_{y}$ is uncountable and closed for some $y \in \mathbb{R}$, then since $\left|H \cap U_{y}\right|<\mathfrak{c}$ and every perfect set can be split into continuum many perfect subsets, there exists a perfect set $P^{\prime} \subseteq P \cap U_{y}$ disjoint from $H$.

On the other hand, if there is no such $y$ then $P=B_{\alpha}$ for some $\alpha$ and therefore $|P \cap H|<\mathfrak{c}$. Thus again by splitting $P$ into continuum many pairwise disjoint perfect subsets, there must be a perfect subset of $P$ disjoint from $H$.

THEOREM 10. There exists a Hamel basis, H, for the reals which is a Marczewski null set.

Obviously, this implies Theorem 8 , since $(H \times\{0\}) \cup(\{0\} \times H)$ is a Marczewski null Hamel basis for the plane. But the proof is a little messier so we chose to do the one for the plane first.

For $p, q \in{ }^{\omega} 2$ define

$$
\sigma(p, q)=\sum_{n=0}^{\infty} \frac{p(n)}{2^{2 n+1}}+\sum_{n=0}^{\infty} \frac{q(n)}{2^{2 n+2}} .
$$

So we are basically looking at the even and odd digits in the binary expansion. The function $\sigma(p, q)$ maps ${ }^{\omega} 2 \times{ }^{\omega} 2$ onto the unit interval $[0,1]$. For any $p \in{ }^{\omega} 2$ define

$$
U_{p}=\left\{\sigma(p, q): q \in{ }^{\omega} 2\right\}
$$

The following is the analogue of Lemma 9 .

Lemma 11. Suppose we have a subspace, $V \subseteq \mathbb{R}$, with $|V|<\mathfrak{c}$ and $1 \in V, p \in{ }^{\omega} 2, U \subseteq U_{p}$ with $|U|<\mathfrak{c}$, and $z \in \mathbb{R}$. Then there exists a finite $F \subseteq U_{p} \backslash U$ such that

$$
z \in \operatorname{span}(V \cup F) \quad \text { and } \quad \operatorname{span}(F) \cap V \text { is trivial. }
$$


Proof. CASE 1: $z=\sigma(\underline{0}, q)$ (where $\underline{0} \in \omega_{2}$ is the constantly zero function).

We may assume that there are infinitely many $n$ such that $q(n)=0$, because otherwise $z \in \mathbb{Q}$ and so we may take $F$ to be empty. Let

$$
A=\{n: q(n)=0\} \text {. }
$$

For any $B \subseteq A$ define the pair $q_{B}, q_{B}^{\prime} \in{ }^{\omega} 2$ as follows:

$$
q_{B}(n)=\left\{\begin{array}{ll}
q(n) & \text { if } n \notin B, \\
1 & \text { if } n \in B,
\end{array} \quad q_{B}^{\prime}(n)= \begin{cases}0 & \text { if } n \notin B, \\
1 & \text { if } n \in B .\end{cases}\right.
$$

Since $q(n)=0$ for each $n \in B$, it follows that $q(n)=q_{B}(n)-q_{B}^{\prime}(n)$ for every $n$. Since we never do any "borrowing" we have

$$
z=\sigma(\underline{0}, q)=\sigma\left(p, q_{B}\right)-\sigma\left(p, q_{B}^{\prime}\right) .
$$

Since $|U|<\mathfrak{c}$ there are continuum many $B \subseteq A$ such that neither $\sigma\left(p, q_{B}\right)$ nor $\sigma\left(p, q_{B}^{\prime}\right)$ are in $U$. Fix one of these $B$ 's and let

$$
F \subseteq\left\{\sigma\left(p, q_{B}\right), \sigma\left(p, q_{B}^{\prime}\right)\right\} \subseteq U_{p} \backslash U
$$

be minimal such that $z \in \operatorname{span}(V \cup F)$.

CASE 2: $z=\sigma(q, \underline{0})$. Since

$$
\frac{1}{2} z=\frac{1}{2} \sigma(q, \underline{0})=\sigma(\underline{0}, q)
$$

this follows easily from Case 1 .

To prove the result for general $z \in \mathbb{R} \backslash \mathbb{Q}$ first we may assume that $z=\sigma\left(q_{1}, q_{2}\right)$ for some $q_{1}, q_{2} \in{ }^{\omega} 2$ since a rational multiple of $z$ is in $[0,1]$. Next we may apply Case 1 to $\sigma\left(\underline{0}, q_{2}\right)$ and then iteratively (as in the proof of Lemma 9) to $\sigma\left(q_{1}, \underline{0}\right)$. Then since $z=\sigma\left(q_{1}, \underline{0}\right)+\sigma\left(\underline{0}, q_{2}\right)$ the lemma is proved.

Proof of Theorem 10. For any distinct $p_{1}, p_{2} \in \omega_{2}$ if neither is eventually one, then $U_{p_{1}}$ and $U_{p_{2}}$ are disjoint. The proof is now similar to that of Theorem 8 , using the family of $U_{p}$ for $p \in{ }^{\omega} 2$ which are not eventually one.

REMARK 12. Similar proofs can be given to produce Marczewski null Hamel bases for $\mathbb{R}^{n}, \mathbb{Q}^{\omega}$, and $\mathbb{R}^{\omega}$. For $\mathbb{R}^{n}$ one can either modify the proofs of Theorem 8 and Lemma 9 , or else observe (for example when $n=3$ ) that if $H$ is a Marczewski null Hamel basis for $\mathbb{R}$, then

$$
(H \times\{0\} \times\{0\}) \cup(\{0\} \times H \times\{0\}) \cup(\{0\} \times\{0\} \times H)
$$

is a Marczewski null Hamel basis for $\mathbb{R}^{3}$. If $X=\mathbb{Q}^{\omega}$ or $X=\mathbb{R}^{\omega}$ then $X$ is isomorphic to $X \times X$ and the proofs are similar to the proof for the plane.

Conjecture 13. Suppose $X$ is an uncountable completely metrizable separable metric space which is also a vector space over a field $\mathbb{F}$ and scalar 
multiplication and vector sum are Borel maps. Then there exists a basis $H$ for $X$ over $\mathbb{F}$ such that $H$ is Marczewski null.

Note that our conjecture reduces to the case where the field $\mathbb{F}$ is either $\mathbb{Q}$ or $\mathbb{Z}_{p}$ for some prime $p$. This is because if $\mathbb{K}$ is a subfield of $\mathbb{F}$ and and $H$ is a Marczewski null basis for $X$ over $\mathbb{K}$, then some maximal linearly independent (over $\mathbb{F}$ ) subset of $H$ is a Marczewski null basis for $X$ over $\mathbb{F}$.

F. B. Jones [4] constructed a Hamel basis containing a perfect set and attributed the construction of what might be called Vitali-independent perfect set to R. L. Swain.

TheOREM 14. There is a Hamel basis for $\mathbb{R}$ which is Marczewski measurable and perfectly dense.

Pr o of. Let $C$ be a linearly independent Cantor set and $H_{0}$ a Marczewski null Hamel basis. Split $C$ into countably many Cantor sets $C_{0}, C_{1}, \ldots$, fix a basis $\left\{B_{n}: n<\omega\right\}$ for the topology of the real line and for each $n$ pick a non-zero rational $q_{n}$ such that $q_{n} C_{n}$ intersects $B_{n}$. Note that

$$
C^{\prime}=\bigcup\left\{q_{n} C_{n}: n<\omega\right\}
$$

is still linearly independent (though not a Cantor set) and for all open sets $U$ there exists a perfect $P \subseteq C^{\prime} \cap U$. Let $H_{1} \subseteq H_{0}$ be maximal such that

$$
H=C^{\prime} \cup H_{1}
$$

is linearly independent. It is easy to see that $H$ works.

Borel additive mappings. We might hope to obtain Theorem 10 as a corollary to Theorem 8 getting a Borel linear isomorphism between $\mathbb{R} \times \mathbb{R}$ and $\mathbb{R}$. Since a Borel bijection preserves the Marczewski null sets, we would be able to obtain a Marczewski null Hamel basis for the reals from one for the plane.

This will not work because of the following result. A mapping is called additive iff $f(x+y)=f(x)+f(y)$ for any $x$ and $y$. Note that if $f$ is additive, then $f(r x)=r f(x)$ for any rational $r$.

Theorem 15. Any additive Borel map $f: \mathbb{R} \times \mathbb{R} \rightarrow \mathbb{R}$ fails to be one-toone.

Lemma 16. Suppose $g: \mathbb{R} \rightarrow \mathbb{R}$ is an additive Borel map. Then there exists a comeager $G \subseteq \mathbb{R}$ and a real a such that $g(x)=$ ax for every $x \in G$.

Proof. This is due to F. Burton Jones [4]. Since $g$ is additive it is not hard to prove that $g(a x)=a g(x)$ for every rational $a \in \mathbb{Q}$ and real $x$. Also, since $g$ is Borel there exists a comeager $G$ such that the restriction of $g$ to $G$ is continuous. Since $a G$ is comeager for any non-zero $a$ we may without loss assume that $a G \subseteq G$ for every non-zero rational $a$. Let $x_{0}$ be 
any fixed non-zero element of $G$. For any $a \in \mathbb{Q}$ we have $g\left(a x_{0}\right)=a g\left(x_{0}\right)$ and $a x_{0} \in G$. So by the continuity of $g$ we get $g\left(y x_{0}\right)=y g\left(x_{0}\right)$ for any $y$ with $y x_{0} \in G$. Now for any $x \in G$,

$$
g(x)=g\left(\frac{x}{x_{0}} x_{0}\right)=\frac{x}{x_{0}} g\left(x_{0}\right)=x \frac{g\left(x_{0}\right)}{x_{0}}
$$

and so $a=g\left(x_{0}\right) / x_{0}$ works.

Proof of Theorem 15. Assume that $f$ is an additive map. By the lemma there exist comeager $G_{i}$ and reals $a_{i}, i=0,1$, such that for every $x \in G_{0}$ we have $f(x, 0)=a_{0} x$ and for every $y \in G_{1}$ we have $f(0, y)=a_{1} y$. Since $f$ is additive it follows that for every $x, y \in G=G_{0} \cap G_{1}$,

$$
f(x, y)=a_{0} x+a_{1} y \text {. }
$$

If either $a_{i}$ is zero, then of course $f$ is not one-to-one. So assume both are non-zero. Let $x$ and $x^{\prime}$ be arbitrary distinct elements of $G$ and define

$$
z=-\frac{a_{0}}{a_{1}}\left(x-x^{\prime}\right)
$$

Since $G$ is comeager, so is $G+z$ and hence we can choose $y$ in both $G$ and $G+z$. If we let $y^{\prime}$ be so that $y=y^{\prime}+z$, then $y^{\prime}=y-z \in G$ and

$$
f(x, y)=a_{0} x+a_{1} y=a_{0} x+a_{1} y^{\prime}-a_{0}\left(x-x^{\prime}\right)=a_{0} x^{\prime}+a_{1} y^{\prime}=f\left(x^{\prime}, y^{\prime}\right)
$$

and $f$ is not one-to-one.

We use similar Baire category arguments to prove the following theorem:

THEOREM 17. There is no Borel (or even Baire) 1-1 additive function $f$ of the following form for any $n=1,2, \ldots$ :

(1) $f: \mathbb{R}^{n+1} \rightarrow \mathbb{R}^{n}$,

(2) $f: \mathbb{R}^{n} \rightarrow \mathbb{Q}^{\omega}$, or $f: \mathbb{R}^{n} \rightarrow \mathbb{Z}^{\omega}$ (even for any 1-1 additive $f$ ),

(3) $f: \mathbb{Q}^{\omega} \rightarrow \mathbb{R}^{n}$, or $f: \mathbb{Z}^{\omega} \rightarrow \mathbb{R}^{n}$.

Proof. (1) This argument is a generalization of Theorem 15. There exists a comeager $G \subseteq \mathbb{R}$ and a linear transformation $L: \mathbb{R}^{n+1} \rightarrow \mathbb{R}^{n}$ with the property that

$$
f\left(x_{1}, \ldots, x_{n+1}\right)=L\left(x_{1}, \ldots, x_{n+1}\right) \quad \text { for any } x_{1}, \ldots, x_{n+1} \in G .
$$

Since $L$ cannot be $1-1$ there must be distinct vectors $u$ and $v$ with $L(u)=$ $L(v)$. Since $G$ is comeager there exists a vector $w$ such that $u_{i}+w_{i}, v_{i}+w_{i}$ $\in G$ for all coordinates $i=1, \ldots, n+1$ (choose $\left.w_{i} \in\left(G-u_{i}\right) \cap\left(G-v_{i}\right)\right)$. But then

$f(u+w)=L(u+w)=L(u)+L(w)=L(v)+L(w)=L(v+w)=f(v+w)$ implies that $f$ is not $1-1$.

(2) It is enough to prove this for the case $f: \mathbb{R}^{1} \rightarrow \mathbb{Q}^{\omega}$, since there are such maps from $\mathbb{R}^{1}$ into $\mathbb{R}^{n}$ and from $\mathbb{Z}^{\omega}$ into $\mathbb{Q}^{\omega}$. Let $f(x)(m) \in \mathbb{Q}$ refer to 
the $m$ th coordinate of $f(x)$. If $f$ is 1-1 and additive, then for each non-zero $x \in \mathbb{R}$ there is some $m$ such that $f(x)(m) \neq 0$. By Baire category there must exist some $q_{0} \in \mathbb{Q}$ with $q_{0} \neq 0$, coordinate $m$, open interval $I$ and $H \subseteq I$ comeager in $I$ such that

$$
f(x)(m)=q_{0} \quad \text { for every } x \in H .
$$

But this is impossible because we can find $\varepsilon \in \mathbb{Q}$ with $\varepsilon$ close to 1 but different from 1 and some $x$ such that $x, \varepsilon x \in H$ but

$$
f(x)+f(\varepsilon x)=f(x+\varepsilon x)=f((1+\varepsilon) x)=(1+\varepsilon) f(x) .
$$

Since both $x$ and $\varepsilon x$ are in $H$ we have $f(x)(m)=f(\varepsilon x)(m)=q_{0}$, contradicting $2 q_{0} \neq(1+\varepsilon) q_{0}$.

(3) We show there is no such map $f: \mathbb{Z}^{\omega} \rightarrow \mathbb{R}^{n}$. Since there is a 1-1 additive Borel map (inclusion) from $\mathbb{Z}^{\omega}$ into $\mathbb{Q}^{\omega}$, this suffices. We start by giving the proof for $n=1$. Assume for contradiction that $G \subseteq \mathbb{Z}^{\omega}$ is a comeager $G_{\delta}$-set and $f\lceil G$ is continuous on $G$.

The topology on $\mathbb{Z}^{\omega}$ is determined by the basic open sets

$$
[s]=\left\{x \in \mathbb{Z}^{\omega}: s \subseteq x\right\}
$$

where $s \in \mathbb{Z}^{<\omega}$ is the set of finite sequences from $\mathbb{Z}$.

Claim. For any $N \in \omega$ and any $s \in \mathbb{Z}^{<\omega}$ there exists $t \in \mathbb{Z}^{<\omega}$ with $s \subseteq t$ and for every $x \in G \cap[t]$ we have $f(x)>N$.

Proof. Let $m=|s|$ be the length of $s$ (so $s=\langle s(0), \ldots, s(m-1)\rangle$ ). For each $k \in \mathbb{Z}$ let $x_{k} \in \mathbb{Z}^{\omega}$ be the sequence which is all zeros except on the $m$ th coordinate where it is $k$. Since $f$ is additive and 1-1 we must have either $\lim _{k \rightarrow \infty} f\left(x_{k}\right)=\infty$ or $\lim _{k \rightarrow-\infty} f\left(x_{k}\right)=\infty$. Since $G$ is comeager there exists $u \in[s]$ such that $u+x_{k} \in G$ for every $k \in \mathbb{Z}$ (i.e., choose $\left.u \in \bigcap_{k \in \mathbb{Z}}\left(-x_{k}+G\right)\right)$. Note that $u+x_{k} \in[s]$ for every $k$ and $f\left(u+x_{k}\right)=$ $f(u)+f\left(x_{k}\right)$, hence for some $k \in \mathbb{Z}$ we have $f\left(u+x_{k}\right)>N$. Since $f$ is continuous on $G$ we can find the $t$ as required. This proves the Claim.

According to the Claim for each $N$ there exists a dense open set $D_{N}$ such that for every $x \in D_{N} \cap G$ we have $f(x)>N$. But this is a contradiction since it implies

$$
G \cap \bigcap_{N \in \omega} D_{N}=\emptyset .
$$

For the case of $f: \mathbb{Z}^{\omega} \rightarrow \mathbb{R}^{n}$ the argument is similar, we just prove a claim that says: For any $N \in \omega$ and any $s \in \mathbb{Z}^{<\omega}$ there exists $t \in \mathbb{Z}^{<\omega}$ with $s \subseteq t$ and for every $x \in G \cap[t]$ we have $f(x)(i)>N$ for some coordinate $i<n$. 


\section{References}

[1] C. Burstin, Die Spaltung des Kontinuums in $\mathfrak{c}$ in Lebesgueschem Sinne nichtmessbare Mengen, Sitzungsber. Akad. Wiss. Wien Math. Nat. Klasse Abt. IIa 125 (1916), 209-217.

[2] P. Erdős, On some properties of Hamel bases, Colloq. Math. 10 (1963), 267-269.

[3] J. Feldman and C. C. Moore, Ergodic equivalence relations, cohomology, and von Neumann algebras I, Trans. Amer. Math. Soc. 234 (1977), 289-324.

[4] F. B. Jones, Measure and other properties of a Hamel basis, Bull. Amer. Math. Soc. 48 (1942), 472-481.

[5] A. S. Kechris, Classical Descriptive Set Theory, Grad. Texts in Math. 156, Springer, 1995.

[6] M. Kuczma, An introduction to the theory of functional equations and inequalities. Cauchy's equation and Jensen's inequality, Prace Nauk. Uniw. Ślạsk. 489, Uniw. Ślaski, Katowice, and PWN, Warszawa, 1985.

[7] A. W. Miller, Special subsets of the real line, in: Handbook of Set-Theoretic Topology, K. Kunen and J. E. Vaughan (eds.), Elsevier, 1984, 201-233.

[8] W. Sierpiński, Sur la question de la mesurabilité de la base de Hamel, Fund. Math. 1 (1920), 105-111.

[9] J. H. Silver, Counting the number of equivalence classes of Borel and coanalytic equivalence relations, Ann. Math. Logic 18 (1980), 1-28.

[10] E. Szpilrajn (Marczewski), Sur une classe de fonctions de M. Sierpiński et la classe correspondante d'ensembles, Fund. Math. 24 (1935), 17-34.

Department of Mathematics

University of Wisconsin-Madison

Van Vleck Hall

480 Lincoln Drive

Madison, WI 53706-1388, U.S.A.

E-mail: miller@math.wisc.edu

Web: http://www.math.wisc.edu/ miller/
Institute of Mathematics Bulgarian Academy of Sciences Acad. G. Bontchev street, bl. 8 1113 Sofia, Bulgaria E-mail: sgpopv@bgcict.acad.bg

Department of Mathematics Auburn University 218 Parker Hall

Auburn, AL 36849-5310, U.S.A. E-mail: popvast@mail.auburn.edu Web: http://www.auburn.edu/ popvast/

Received 6 December 1999;

in revised form 24 August 2000 